\title{
Critical analysis of ICU/HC beds in South Africa: 2008-2009
}

\author{
K Naidoo, ${ }^{1}$ MB ChB, MMed (FamMed), LLM (Medical Law); J Singh, ${ }^{2}$ BA, LLB, LLM, MHSc, PhD; \\ U Lalloo, ${ }^{3} \mathrm{MB}$ ChB, FCP, MD, DOH, FCCP, FRCP

\begin{abstract}
${ }^{1}$ Department of Family Medicine, School of Nursing and Public Health, Nelson R Mandela School of Medicine, University of KwaZulu-Natal, Durban, South Africa

${ }^{2}$ Centre for the AIDS Programme of Research in South Africa (CAPRISA), Doris Dukes Medical Centre Research Institute, Nelson R Mandela School of Medicine, University of KwaZulu-Natal, Durban, South Africa

${ }^{3}$ Department of Pulmonology and Critical Care, School of Clinical Medicine, Nelson R Mandela School of Medicine, University of KwaZulu-Natal, Durban, South Africa
\end{abstract}

Corresponding author: U Lalloo (umeshlalloo@gmail.com)

\begin{abstract}
Objective. To determine the national distribution of intensive care unit (ICU)/high care (HC) beds and the implications for ICU bed availability in the envisaged national health insurance (NHI) scheme.

Methods. A descriptive, non-interventional, observational study design was used. A desk-top audit of all public and private sector ICUs, including ICU/HC beds, in South Africa was undertaken for the period 2008 - 2009. For this study, both categories were analysed and referred to as ICU beds, as they reflect the critical care component of the health service in South Africa.

Results. In 2008 - 2009, there were 4719 ICU beds in the private and public sectors in South Africa, with 75\% (3 533) in the former and 25\% (1 186) in the latter. The majority of ICU beds in the two sectors were located in three provinces: Gauteng (49\%), KwaZulu-Natal (14\%) and Western Cape (15\%), representing 78\% of ICU beds (3 703/4 719) and catering for $54 \%$ of the country's population. Eastern Cape had fewer than 300 beds, North West and Mpumalanga had fewer than 150 beds, and Limpopo and Northern Cape had 66 and 47 beds respectively. With the proposed NHI scheme, the number of ICU beds available would be 4719 , which would translate into a bed:population ratio of approximately 1:10 000. However, there are large variations across the provinces, which makes the availability of this level of care in some provinces non-existent.

Conclusion. While increasing the number of ICU beds in the public sector will open critical care services to more users, the NHI scheme would not solve the huge discrepancies of access to ICUs, and availability of critical care staff, across the provinces.
\end{abstract}

S Afr Med J 2013;103(10):751-753. DOI:10.7196/SAMJ.6415

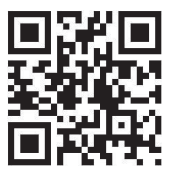

The American College of Emergency Physicians (ACEP) defines a critical illness or injury as one that acutely impairs one or more vital organ systems, resulting in a high probability of imminent or lifethreatening deterioration in the patient's condition. ${ }^{[1]}$ These conditions require critical care services, which are defined as the direct delivery of medical care to a critically ill or injured patient, and involves decisions being made to prevent any further life-threatening deterioration of the patient's condition. ${ }^{[1]}$

Critical care services are rendered by critical care specialists (CCSs) in critical care units, also called ICUs or HCUs, although the level of care in an ICU is invariably greater than that available in an HCU. The ICU enables the CCS team to monitor and care for patients with potentially severe physiological instability that demands highly technological and/or artificial life support.

As the population ages, and with new and re-emerging health priorities, such as trauma, HIV/AIDS and tuberculosis that increase morbidity and mortality, there is an expectation that critical care will be available on demand to all who need it. ${ }^{[2]}$ This point raises issues of cost, cost-effectiveness, availability and, moreover, ethical issues relating to rationing of scarce resources and questions regarding medical futility. ${ }^{[2]}$

In South Africa (SA), the $8.6 \%$ of GDP spent on health consists of $4.4 \%$ in the private sector (which provides for $16 \%$ of the population) and $4.2 \%$ in the public sector, which provides for the remaining $84 \%{ }^{[3]}$ Almost 5 times as much money is spent on each person on medical aid as on an uninsured person using the public sector. ${ }^{[4]}$ It is estimated that only $2 \%$ of the uninsured population would access private healthcare. ${ }^{[5]}$ The public sector is underresourced relative to the population that it serves and the burden of disease presenting to it. It also has disproportionately less human resources than the private sector, hence the move in SA towards a national health insurance (NHI) scheme that would enable patients to access equitable, appropriate and high-quality health services, regardless of their socio-economic status. ${ }^{[3]}$ In recent years, there has been concern over the inefficiencies prevalent in ICU use, ${ }^{[6]}$ lack of guidelines and protocols, discordant use of ICUs, ${ }^{[7]}$ and the high cost of care. ${ }^{[8]}$

Following world-wide trends, the demand for ICU beds for eligible patients exceeds supply, ${ }^{[9]}$ and this form of expensive technology needs to be prioritised for those patients who are most likely to recover. In SA, with its high burden of disease and consequent high demand for such a level of care, CCSs often face ethical dilemmas regarding who might be admitted. The present study was conducted to establish the number of ICU beds available in the private and public sectors and whether the envisaged NHI scheme would make a difference to ICU bed availability.

\section{Methods}

Information from proceedings of the South African Parliamentary Portfolio Committee on Health ${ }^{[10]}$ (accessed electronically), personal correspondence with the Head of Department in the Gauteng Department of Health, and statistics from the Health Annals - SA, $2008,{ }^{[5]}$ an official publication of the Hospital Association of South 
Africa (HASA) representing private hospital groups, was used. A desk-top audit using a descriptive, non-interventional, observational study design was undertaken of all public and private sector ICU/ HCU beds in SA, with data from 2008 and 2009 being used owing to their ready availability. The ICUs analysed included general medical/ coronary/pulmonology, surgical/trauma/burns, neonatal/paediatric, and labour ward/maternity ICUs. Population statistics for 2009 were obtained from Stats SA. ${ }^{[11]}$ Descriptive statistics were used to analyse the data.

\section{Results}

Within the public sector, $23 \%(92 / 396)$ of hospitals had ICUs v. $84 \%$ in the private sector $(216 / 256){ }^{[12]}$ The data presented (Tables 1 - 3) include the total population per province, the number of public sector ICU beds, the number of private sector ICU beds, the total number of ICU beds as a combination of the private and public sectors, and the bed-to-population ratio.

In 2008 - 2009, there were 4719 ICU/HC beds in SA, with 75\% (3 $533)$ in the private sector and 25\% (1 186) in the public sector. The majority of ICU beds were located in 3 provinces: Gauteng (49\%, 2311 beds), KwaZulu-Natal (14\%, 672 beds) and Western Cape (15\%, 719 beds), representing $78 \%$ of all ICU beds (3 703/4 719) and catering for $54 \%$ of the country's population. The Eastern Cape had fewer than 300 beds, North West and Mpumalanga had fewer than 150 beds, and Limpopo and Northern Cape had 66 and 47 beds respectively.

The population distribution significantly affected the bed: population ratio. The public sector bed:population ratio in the Western Cape was approximately 1:20 000 and in Gauteng, the most populous province, 1:25 000, and in the Free State 1:30 000. KwaZuluNatal, which comprises $21 \%$ of the population and carries the highest burden of HIV/AIDS, has a bed: population ratio of 1:45 000. The ratio for the rest of the provinces ranges from 1:50 000 to 1:150 000 (Table 3).

\section{Discussion}

Within the public sector, 23\% of hospitals have ICUs (92/396) compared with $84 \%$ in the private sector $(216 / 256) .{ }^{[12]}$ With the proposed NHI scheme, ${ }^{[3]}$ the total ICU bed availability to the country would be 4719 beds, which would translate to a bed:population ratio of approximately 1:10 000, assuming that all beds are commissioned. The NHI scheme would not solve the huge discrepancies of access to ICU beds across the provinces. Bhagwanjee and Scribante, in their 2005 'National audit', concluded

Table 1. ICU/HC beds in South Africa 2008 - 2009

\begin{tabular}{|c|c|c|c|c|c|c|}
\hline \multirow[b]{2}{*}{ Province } & \multicolumn{2}{|c|}{ Population } & \multicolumn{2}{|c|}{ Public } & \multicolumn{2}{|c|}{ Private } \\
\hline & $N$ (million) (\%) & $\%$ of total & Beds $(N)$ & $\%$ of total & Beds $(N)$ & $\%$ of total \\
\hline EC & 6.648 & 13.5 & 90 & 6.9 & 162 & 4.6 \\
\hline FS & 2.902 & 5.9 & 103 & 7.9 & 293 & 8.2 \\
\hline GT & 10.531 & 21.4 & 397 & 33.5 & 1914 & 54.2 \\
\hline $\mathrm{KZN}$ & 10.449 & 21.2 & 224 & 17.2 & 448 & 12.7 \\
\hline LP & 5.227 & 10.6 & 35 & 2.7 & 31 & 0.9 \\
\hline MP & 3.606 & 7.3 & 40 & 3.1 & 101 & 2.8 \\
\hline NC & 1.147 & 2.3 & 21 & 1.6 & 26 & 0.7 \\
\hline NW & 3.450 & 7 & 32 & 2.5 & 83 & 2.3 \\
\hline WC & 5.356 & 10.9 & 244 & 18.8 & 475 & 13.4 \\
\hline Total & 49.320 & 100 & 1186 & 25 & 3533 & 75 \\
\hline
\end{tabular}

Table 2. Public and private ICU/HC beds as a percentage of the total per province

\begin{tabular}{|c|c|c|c|c|c|c|}
\hline \multirow[b]{2}{*}{ Province } & \multicolumn{2}{|c|}{ Public } & \multicolumn{2}{|c|}{ Private } & \multicolumn{2}{|c|}{ Beds per province } \\
\hline & Beds $(N)$ & $\%$ of total & Beds $(N)$ & $\%$ of total & Total beds & $\%$ of total \\
\hline EC & 90 & 1.9 & 162 & 3.4 & 252 & 5.3 \\
\hline FS & 103 & 2.2 & 293 & 6.2 & 396 & 8.4 \\
\hline GT & 397 & 8.4 & 1914 & 40.6 & 2311 & 49.0 \\
\hline KZN & 224 & 4.8 & 448 & 9.5 & 672 & 14.2 \\
\hline LP & 35 & 0.7 & 31 & 0.7 & 66 & 1.40 \\
\hline MP & 40 & 0.9 & 101 & 2.1 & 141 & 3.0 \\
\hline NC & 21 & 0.5 & 26 & 0.6 & 47 & 1.0 \\
\hline NW & 32 & 0.7 & 83 & 1.8 & 115 & 2.4 \\
\hline WC & 244 & 5.2 & 475 & 10.0 & 719 & 15.2 \\
\hline Total & 1186 & 25.1 & 3533 & 74.9 & 4719 & 100 \\
\hline
\end{tabular}


Table 3. Public sector ICU/HC bed:population ratio in South Africa: 2008 - 2009

\begin{tabular}{llll}
\hline Province & Population (millions) & Public sector ICU/HC beds & Public sector ICU/HC bed:population ratio \\
\hline EC & 6.648 & 90 & $1: 75000$ \\
FS & 2.902 & 103 & $1: 30000$ \\
GT & 10.531 & 397 & $1: 25000$ \\
KZN & 10.449 & 224 & $1: 45000$ \\
LP & 5.227 & 35 & $1: 150000$ \\
MP & 3.606 & 40 & $1: 90000$ \\
NC & 1.147 & 21 & $1: 55000$ \\
NW & 3.450 & 32 & $1: 110000$ \\
WC & 5.356 & 244 & $1: 20000$ \\
Total & 49.320 & 1186 & \\
EC = Eastern Cape; FS = Free State; GT = Gauteng; KZN = KwaZulu-Natal; LP = Limpopo; MP = Mpumalanga; NC = Northern Cape; NW = North West; WC = Western Cape.
\end{tabular}

that in view of huge discrepancies in the availability of ICU beds in the country, there is a compelling need for regionalisation of ICU services in SA. ${ }^{[12]}$ The data presented in our 2008 - 2009 audit would strongly support their statement. These statistics highlight the importance of triage protocols for ICU admission and discharge, and effective treatment guidelines and algorithms in a resourcelimited environment.

It would appear that the resource constraints in SA, together with the burden of HIV infection and the associated complications, impose a greater burden on already limited ICU bed resources, whereas in the USA, it would appear that ad hoc admissions to ICU as a result of a lack of effective implementation of triage policies have contributed to unnecessary ICU admissions. This practice is largely influenced by the threat of litigation by patients and their families. Kohn et al, in their questionnaire survey to CCSs in 2011, concluded: 'this study suggests that many ICU clinicians feel compelled to provide salvage critical care for identifiable patients with grave prognoses even when doing so measurably contravenes society's interests..[13]

\section{Human resources}

In the 2007 National Audit of Critical Care Services, commissioned by the Critical Care Society of Southern Africa, it was noted that there was a shortage of approximately 291 CCSs in SA, which at current rates of production would take 30 years to establish. ${ }^{[12]}$ Notably, provinces such as Limpopo, Mpumalanga, Northern Cape and North West have no CCSs. In KZN overall, $19 \%$ of ICUs are staffed by CCSs. ${ }^{[12]}$ In addition, patient autonomy combined with a biased feefor-service private healthcare sector will make access to ICUs much easier in the private than in the public health sector. ${ }^{[14]}$

\section{Conclusion}

Innovative changes such as the implementation of fast-track anaesthesia and enhanced ICU capabilities; ${ }^{[15]}$ the development of sub-intensive, intermediate and step-down units; ${ }^{[16]}$ and the increased use of non-invasive ventilation in regular wards, rather than only in ICUs, ${ }^{[17]}$ will potentially decrease the need for ICU beds, which are always in demand and in short supply. Such changes will have the desired effect also of decreasing the need for CCSs in favour of expansion of hospitalists/generalists in number and scope of practice. ${ }^{[18]}$ The need for ICU care would be effectively decreased, as also would be hospital length of stay and costs. ${ }^{[8]}$ Triage tools, based on scientifically validated guidelines and protocols, including the use of admission and discharge criteria, are required to enable optimal utilisation of intensive care services in a limited-resource environment such as SA.

The NHI scheme cannot be expected to solve the huge discrepancies of access to ICU beds across SA.

\section{References}

1. American College of Emergency Physicians. http://www.acep.org/ (accessed 26 February 2012).

2. A critical look at critical care (editorial). Lancet;2010;376(9749):1273. [http://dx.doi.org/10.1016/ s0140-6736(10)61896-x]

3. National Health Insurance in South Africa Policy Paper. http://www.info.gov.za/view/ DownloadFileAction?id $=148470$ (accessed 25 February 2012).

4. Harrison D. An Overview of Health and Health Care in South Africa 1994 - 2010: Priorities, Progress and Prospects for New Gains commissioned by the Henry J Kaiser Family Foundation. http://www. doh.gov.za/docs/reports/2010/overview1994-2010.pdf (accessed 25 February 2012).

5. Hospital Association of South Africa. Health Annals 2008. http://www.hasa.co.za/media/uploads/ documents/file/2009-02-26/On_the_Brink_2.pdf (accessed 20 March 2012).

6. Angus DC. Caring for the critically ill patient: Challenges and opportunities. JAMA 2007;298(4):456-458. . Luce JM, Rubenfeld GD. Can health care costs be reduced by limiting intensive care at the end of life? Am J Respir Crit Care Med 2002;165(6):750-754

8. Halpern NA, Pastores SM. Critical care medicine in the United States 2000-2005: an analysis of bed numbers, occupancy rates, payer mix, and costs. Crit Care Med 2010;38(1):65-71. [http://dx.doi. org/10.1097/CCM.0b013e3181b090d0]

9. Simchen E, Sprung CL, Galai N, et al. Survival of critically ill patients hospitalized in and out of 9. Simchen E, Sprung CL, Galai N, et al. Survival of critically ill patients hospitalized in and out
intensive care units under paucity of intensive care unit beds. Crit Care Med 2004;32(8):1654-1661.

10. Question \& Replies No. 2071 to 2100 - Number of ICU BEDS in SA Public Hospitals by Parliamentary Monitoring Group. http://www.pmg.org.za/node/19587 (accessed 21 February 2012).

11. Statitics South Africa. Statistical Release P0302-2010. http://www.statssa.gov.za/publications/P0302/ P03022010.pdf (accessed 24 February 2012)

12. Bhagwanjee S, Scribante J. National audit of critical care resources in South Africa - unit and bed distribution. S Afr Med J 2007;97(12):1311-1314

13. Kohn R, Rubenfeld G, Levy M, Ubel P, Halpern S. Rule of rescue or the good of the many? An analysis of physicians' and nurses' preferences for allocating ICU beds. Intensive Care Med 2011;37(7):12101217. [http://dx.doi.org/10.1007/s00134-011-2257-6]

14. Osborne M, Patterson J. Ethical allocation of ICU resources:a view from the USA. Intensive Care Med 1996;22(10):1010-1014.

15. Flynn M, Reddy S, Shepherd W, et al. Fast-tracking revisited: Routine cardiac surgical patients need minimal intensive care. Eur J Cardiothorac Surg 2004;25(1):116-122.

6. Ranhoff AH, Rozzini R, Sabatini T, et al. Subintensive care unit for the elderly: A new model of care for critically ill frail elderly medical patients. Intern Emerg Med 2006;1(3):197-203.

17. Hill NS. Where should noninvasive ventilation be delivered? Respir Care 2009;54(1):62-70

18. Wachter RM. The state of hospital medicine in 2008. Med Clin North Am 2008;92(2):265-273. [http:// dx.doi.org/10.1016/j.mcna.2007.10.008

Accepted 14 November 2012. 